\title{
KECK AND EUROPEAN SOUTHERN OBSERVATORY VERY LARGE TELESCOPE VIEW OF THE SYMMETRY OF THE EJECTA OF THE XRF/SN 2006aj ${ }^{1}$
}

\author{
Paolo A. Mazzali, $, 2,4,5$ Ryan J. Foley, ${ }^{6}$ Jinsong Deng, ${ }^{4,5,7}$ Ferdinando Patat, ${ }^{8}$ Elena Pian, ${ }^{3}$ Dietrich Baade, ${ }^{8}$ \\ Joshua S. Bloom, ${ }^{6}$ Alexei V. Filippenko, ${ }^{6}$ Daniel A. Perley, ${ }^{6}$ Stefano Valenti,,${ }^{8,9}$ Lifan Wang, ${ }^{10,11}$ \\ Kojt Kawabata, ${ }^{12}$ Keirchi Maeda, ${ }^{13}$ and Ken-IChi Nomoto 4,5 \\ Received 2006 December 15; accepted 2007 March 3
}

\begin{abstract}
Nebular-phase spectra of SN 2006aj, discovered in coincidence with XRF 060218, were obtained with Keck in 2006 July and VLT in 2006 September. At the latter epoch spectropolarimetry was also attempted, yielding a polarization upper limit of $\sim 2 \%$. The spectra show strong [O I] and $\mathrm{Mg}$ I] emission lines, as expected in Type Ic supernovae, but weak Ca II lines. The [Fe II] lines that were strong in SN $1998 \mathrm{bw}$ are much weaker in SN 2006aj, consistent with its lower luminosity. The outer velocity of the line-emitting region is $\sim 8000 \mathrm{~km} \mathrm{~s}^{-1}$ in July and $\sim 7400 \mathrm{~km} \mathrm{~s}^{-1}$ in September, consistent with the relatively low expansion kinetic energy of SN 2006aj. All lines have similar width, and their profiles indicate that no major asymmetries are present in the ejecta at velocities below $v<8000 \mathrm{~km} \mathrm{~s}^{-1}$, except perhaps in the innermost part. The spectra were modeled with a non-LTE code. The mass of ${ }^{56} \mathrm{Ni}$ required to power the emission is $\sim 0.20 M_{\odot}$, confirming earlier results based on the light curve. The oxygen mass is $\sim 1.5 M_{\odot}$, again much less than in SN $1998 \mathrm{bw}$, but $\sim 0.7 M_{\odot}$ larger than the value derived from the early-time modeling. The ejected mass below $8000 \mathrm{~km} \mathrm{~s}^{-1}$ is $\sim 2 M_{\odot}$, confirming that SN 2006aj was only about twice as massive and energetic as the normal Type Ic SN 1994I. The presence of a dense inner core, containing $\sim 1 M_{\odot}$ of mostly oxygen and carbon, is inferred, as in all broad-lined SNe Ic. This core, which may be disklike, is too deep to influence the early light curve and too small to affect the late polarization spectrum.
\end{abstract}

Subject headings: gamma rays: bursts - nuclear reactions, nucleosynthesis, abundances supernovae: general — supernovae: individual (SN 2006aj)

Online material: color figures

\section{INTRODUCTION}

The connection between some gamma-ray bursts (GRBs) and supernovae $(\mathrm{SNe})$ is now well established (see Woosley \& Bloom 2006 for a recent review). The first observational clue for a connection came with the discovery of a bright SN coincident in time and space with GRB 980425 (Galama et al. 1998; Pian et al. 2000). The SN was eventually classified as a Type Ic (for a definition of the class, see Filippenko 1997), indicating an origin from a massive star that lost both the $\mathrm{H}$ and $\mathrm{He}$ envelopes prior to core collapse. SN 1998bw was an exceptional SN Ic, in being very luminous and showing a broad-lined spectrum, indicative of a large kinetic energy of expansion. Such objects have been

\footnotetext{
1 Based on observations made with ESO telescopes at the Paranal and La Silla Observatories under program 277.D-5039(A).

2 Max-Planck-Institut für Astrophysik, 85748 Garching, Germany.

3 National Institute for Astrophysics-OATs, 34143 Trieste, Italy.

4 Department of Astronomy, School of Science, University of Tokyo, Bunkyo-ku, Tokyo 113-0033, Japan.

5 Research Center for the Early Universe, School of Science, University of Tokyo, Bunkyo-ku, Tokyo 113-0033, Japan.

6 Department of Astronomy, University of California, Berkeley, CA 947203411 .

7 National Astronomical Observatories, CAS, Chaoyang District, Beijing 100012, China.

8 European Southern Observatory, 85748 Garching, Germany.

9 Department of Physics, University of Ferrara, 44100 Ferrara, Italy.

${ }^{10}$ Physics Department, Texas A\&M University, College Station, TX 778434242.

${ }^{11}$ Purple Mountain Observatory, Chinese Academy of Sciences, Nanjing, Jiangsu 210008, China.

${ }^{12}$ Hiroshima Astrophysical Science Center, Hiroshima University, Hiroshima 739-8526, Japan.

13 Department of Earth Science and Astronomy, Graduate School of Arts and Science, University of Tokyo, Meguro-ku, Tokyo 153-8892, Japan.
}

called hypernovae (Iwamoto et al. 1998) or broad-lined SNe Ic (SNe Ic-BL; see Woosley \& Bloom 2006). Modeling confirmed the large kinetic energy, $\sim(10-30) \times 10^{51} \mathrm{ergs}$, and showed that the ejected mass was also quite large $\left(\sim 10 M_{\odot}\right.$; Iwamoto et al. 1998 ), pointing to a progenitor star with zero-age main sequence (ZAMS) mass $\sim 40 M_{\odot}$.

Later, other nearby long-duration soft-spectrum GRBs, in the same high-energy class as GRB 980425, were also seen to accompany hypernovae/SNe Ic-BL, confirming the connection (Stanek et al. 2003; Malesani et al. 2004). The SNe had properties very similar to those of SN $1998 \mathrm{bw}$, although the properties of the GRBs were quite different (Mazzali et al. 2003, 2006a). In a popular model, both the SN and the GRB are produced when the rapidly rotating core of a massive star collapses to form a black hole (MacFadyen \& Woosley 1999). The coincidence of a very aspherical event, the GRB, and a SN motivates the search for signatures of asphericity in the $\mathrm{SN}$ as well.

In the case of SN 1998bw, evidence of asphericity came from the nebular spectra. These showed strong emission lines of both [O I ] $\lambda \lambda 6300,6363$ and [Fe II] (a blend near $5200 \AA$ ), as could be expected from a bright SN Ic, but with the peculiarity that even after accounting for blending the [Fe II] lines were broader than the $\left[\mathrm{O}_{\mathrm{I}}\right]$ line, indicating a higher expansion velocity of Fe than of $\mathrm{O}$. This was explained in the context of an axisymmetric explosion, where the Fe produced by the decay of ${ }^{56} \mathrm{Ni}$ is ejected at high velocities near the direction of the GRB jet while oxygen, which is mostly not a product of the explosion but rather left over from the progenitor, is ejected at lower velocities nearer the equator (Mazzali et al. 2001). Maeda et al. (2002) supported this scenario with two-dimensional explosion models and threedimensional synthetic nebular spectra, and suggested that our viewing angle was $\sim 15^{\circ}-30^{\circ}$ from the jet axis (see also Maeda 
et al. 2006). This may be partly responsible for the weakness of GRB 980425 (Ramirez-Ruiz et al. 2005).

In the case of SN $2003 \mathrm{dh} / \mathrm{GRB} 030329$, polarization levels of $\sim 2 \%$ were observed in the first $2-3$ days after the GRB, but they were probably measurements of the GRB afterglow (Kawabata et al. 2003; Greiner et al. 2003 and references therein), dominated by the synchrotron process, which imposes partial polarization. Later polarization measurements, which should not have been affected by the afterglow, suggested that the polarization was small $(P<1 \%)$, although the alignment of the polarization angle suggests that the polarization is nonzero (Kawabata et al. 2003). This could be consistent with a jetlike explosion viewed very close to the jet axis, as the strength of GRB 030329 may suggest.

A later confirmation that the ejecta of bright $\mathrm{SNe}$ Ic can be very aspherical came from the detection of the double-peaked emission profile of the [O I] $\lambda \lambda 6300,6363$ line in SN 2003jd (Mazzali et al. 2005). The profile could be reproduced assuming that we observed an explosion similar to that of SN 1998bw from close to the equatorial plane. A GRB may or may not (Soderberg et al. 2006a) have been produced, and if it was it may not have been detected because it was not pointing toward the Earth.

Asphericity may indeed be a common feature of core-collapse SNe (Leonard et al. 2006), and it is probably stronger in the carbon-oxygen cores, since envelope-stripped SNe show a higher degree of polarization (Wang et al. 1996; Filippenko \& Leonard 2004; Leonard \& Filippenko 2005). Spectropolarimetry of the non-GRB, broad-lined SN Ic 2002ap (Kawabata et al. 2002; Leonard et al. 2002; Wang et al. 2003) revealed polarization of up to $\sim 2 \%$ in the $\mathrm{O}_{\text {I }} 27774$ line and in the Ca II IR triplet at early times, suggesting a deviation from sphericity of $\sim 10 \%-20 \%$.

X-ray flashes (XRFs) are a lower energy and softer subclass of long-duration GRBs (Heise et al. 2001). Previous work had indicated a connection between XRFs and SNe (Fynbo et al. 2004; Tominaga et al. 2004; Soderberg et al. 2005), but high-quality spectra of the SNe had not been obtained until the Type Ic SN 2006aj was discovered coincident with XRF 060218, at a red$\operatorname{shift} z=0.03342$ (Pian et al. 2006). Although SN 2006aj showed a broad-lined spectrum, this was not as broad as those of $\mathrm{SNe}$ $1998 \mathrm{bw}$ and $2003 \mathrm{dh}$. Therefore, SN 2006aj could not, prima facie, be considered an extremely energetic SN from early optical spectroscopy and photometry. Radio observations confirmed this (Soderberg et al. 2006b).

SN 2006aj was modeled (Mazzali et al. 2006b) to have synthesized only $\sim 0.2 M_{\odot}$ of ${ }^{56} \mathrm{Ni}$, and to have ejecta only slightly more massive $\left(\sim 2 M_{\odot}\right)$ and energetic $\left(E \approx 2 \times 10^{51}\right.$ ergs $)$ than "normal" SNe Ic. ${ }^{14}$ These parameters are more appropriate for the collapse of a star of $\sim 20 M_{\odot}$ to a neutron star. Mazzali et al. (2006b) suggested that magnetic activity on the surface of the nascent neutron star increased the energy of the explosion and caused the XRF. In this case, the explosion may not be highly aspherical. The absence of a jet break and the behavior of the radio flux led Soderberg et al. (2006b) to estimate a "jet" opening angle of $\gtrsim 50^{\circ}$.

Large polarization ( $\sim 4 \%$ at $3-5$ days) was observed in $\mathrm{SN}$ 2006aj (Gorosabel et al. 2006). As in the case of SN 2003dh, this may have been due to the relativistic jet and the XRF afterglow synchrotron continuum, but as the data have a later epoch than those of SN 2003dh (Kawabata et al. 2003), and the afterglow

\footnotetext{
14 We use the term "normal" to refer to SNe like SN 1994I (Sauer et al. 2006 and references therein), i.e., SNe with explosion energy $\approx 10^{51}$ ergs, although it is by no means clear that this can be called a normal event.
}

was not as strong as in the case of SN $2003 \mathrm{dh}$, an aspherical fast outflow from the outermost SN ejecta may also have been detected. At later epochs, Gorosabel et al. (2006) found that the polarization level dropped to $\sim 1.4 \%$, which they attributed to the host galaxy.

Obviously, it is important to verify these suggestions using nebular spectroscopy. We observed SN 2006aj with the Keck I telescope and the Very Large Telescope (VLT) when it became visible again after solar occultation. In the following, we discuss the observations $(\S 2)$, present the observational results $(\S 3)$ and the models of the nebular spectra $(\S 4)$, and discuss our findings $(\S 5)$.

\section{OBSERVATIONS AND SPECTRAL ANALYSIS}

On 2006 July 26.61 (UT dates are used throughout this paper), SN 2006aj was observed with the $10 \mathrm{~m}$ Keck I telescope on Mauna Kea, Hawaii, equipped with the Low Resolution Imaging Spectrograph (Oke et al. 1995) with a D560 dichroic to separate the blue and red light paths. The seeing was about $0.8^{\prime \prime}$, and a long slit of width $1^{\prime \prime}$ was aligned along a position angle of $-82^{\circ}$. Although the parallactic angle (Filippenko 1982) at that time was $-52^{\circ}$, the atmospheric dispersion was not very significant, given the relatively low air mass (1.3). The blue-side data were taken with the 600/4000 grism, giving a spectral resolution of $3.5 \AA$, while the red-side data were taken with the $400 / 8500$ grating, giving a spectral resolution of $6.4 \AA$. Two 600 s exposures were obtained. Standard CCD processing was accomplished with IRAF. ${ }^{15}$ The data were extracted using the optimal algorithm of Horne (1986). Wavelength calibration was obtained from internal arc lamps, and slight adjustments were derived from night-sky lines. The spectrophotometric standard stars BD +28 4211 and $\mathrm{BD}+174708$ (observed at the parallactic angle), as well as our own IDL routines, were used for flux calibration and telluric absorption removal (Wade \& Horne 1988; Matheson et al. 2000).

On 2006 September 19.33 and 20.36, SN 2006aj was observed with the $8 \mathrm{~m}$ VLT Kueyen of the European Southern Observatory (ESO) at Cerro Paranal, equipped with the Focal Reducer Spectrograph (FORS1; Appenzeller 1998) in polarimetric mode. The observations were performed with the $300 \mathrm{~V}$ grism and a $1.1^{\prime \prime}$ wide slit, aligned along the parallactic angle, giving a dispersion of $\sim 2.6 \AA$ pixel $^{-1}$ and a resolution of $\sim 11 \AA$ at $5500 \AA$ (full width at half-maximum intensity). The wavelength coverage achieved with this setup is $3300-8600 \AA$. At both epochs the SN was observed at four half-wave plate position angles $\left(0^{\circ}\right.$, $22.5^{\circ}, 45^{\circ}$, and $67.5^{\circ}$ ), for a total integration time of $7200 \mathrm{~s}$. The seeing was $1.2^{\prime \prime}$ on September 19 and $1.5^{\prime \prime}$ on September 20.

The data were bias-subtracted, flattened, and wavelengthcalibrated using standard tasks within IRAF. Stokes parameters, linear polarization degree, and position angle were computed using specific routines written by the authors. Finally, polarization bias correction and error estimates were performed following the prescriptions described by Patat \& Romaniello (2006), while the half-wave plate zero-point angle chromatism was corrected using FORS 1 tabulated data. ${ }^{16}$ In order to reduce the noise, the two data sets have been combined and the final Stokes parameters rebinned to $75 \AA$ bins (29 pixels). The signal-to-noise

15 IRAF is distributed by the National Optical Astronomy Observatory, which is operated by the Association of Universities for Research in Astronomy, under contract with the National Science Foundation.

16 See http://www.eso.org/instruments/fors/inst/pola.html. 


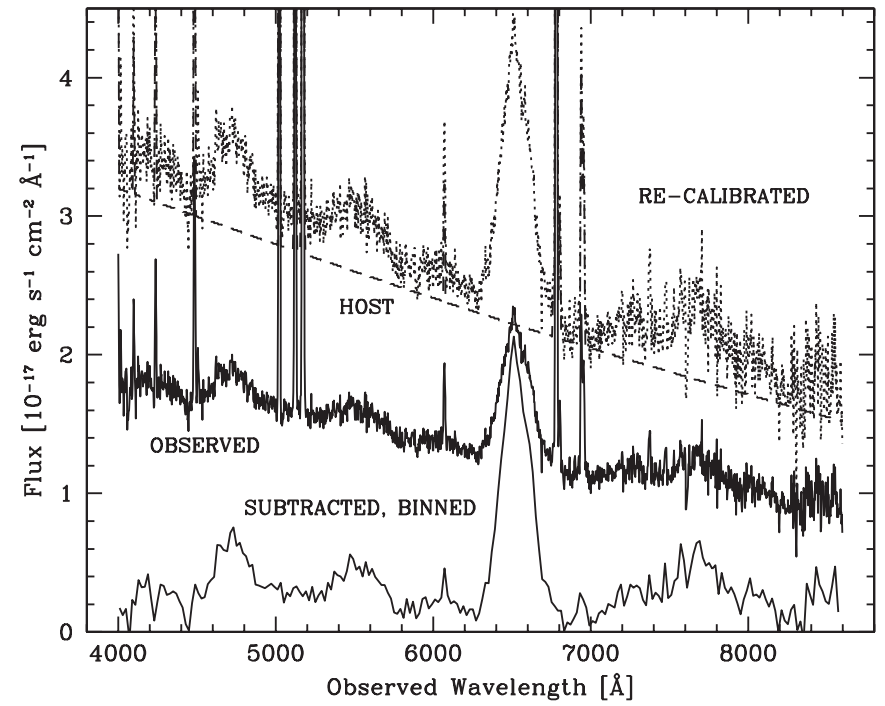

FIG. 1.-VLT FORS1 spectrum of SN 2006aj on 2006 September 19-20. The middle solid curve shows the observed spectrum. The upper thin dotted curve is the observed spectrum recalibrated according to the host galaxy photometry (see $\delta 2$ ). The host galaxy spectrum is shown as a dashed curve. The spectrum of the $\mathrm{SN}$, obtained by subtracting the galaxy spectrum (including the narrow emission lines) from the recalibrated spectrum and binned using a $20 \AA$ boxcar, is the lower solid curve.

ratio $(\mathrm{S} / \mathrm{N})$ in the binned flux spectrum is $\sim 100$ in the $\left[\mathrm{O}_{\mathrm{I}}\right] \lambda \lambda 6300$, 6363 region, corresponding to an expected rms uncertainty in the polarization degree of $\sim 0.7 \%$.

Photometric calibration of the spectra was achieved by observing spectrophotometric standard stars with full polarimetric optics inserted. Instrumental polarization and position angle offset were checked by observing polarized and unpolarized standard stars, within the FORS1 calibration plan.

The total flux spectrum was obtained combining all exposures, giving $\mathrm{S} / \mathrm{N} \approx 30$ in the continuum at $6000 \AA$. At this advanced nebular phase, the only relevant $\mathrm{SN}$ signal is expected to be associated with the nebular emission lines. Therefore, we attributed all observed continuum to the host galaxy. The continuum was evaluated in four spectral regions (near $4400 \AA$, i.e., immediately blueward of the Mg I] line; at 6200 and $7020 \AA$, i.e., on either side of the [O I] line; and at $8170 \AA$ ), and was then interpolated at all wavelengths of our spectral range with a spline and subtracted, along with the host galaxy emission lines, from the observed spectra. We thus obtained the pure SN emission-line spectrum.

In order to compute line intensities the spectral flux was converted to physical units. The SN spectrum is superposed on the host galaxy continuum. Galaxy photometry, however, includes narrow host galaxy emission lines (Cool et al. 2006; Modjaz et al. 2006; Pian et al. 2006; Sollerman et al. 2006). The flux level of the SN spectra is, however, best established by comparing to the galaxy continuum. Therefore, first we estimated from the observed spectra the fractional contribution of the host galaxy emission lines to the galaxy photometry in each filter, and hence determined the photometric level of the galaxy spectral continuum. This allowed us to evaluate the flux level of the observed and subtracted SN spectra, and ultimately to estimate the intensities of the SN nebular emission lines. As the host galaxy of SN 2006aj is very small and compact, the fraction of galaxy and SN light possibly not included in the slit during spectroscopy should be similar, ensuring that our method does not substantially underestimate the galaxy contribution. Figure 1 shows the

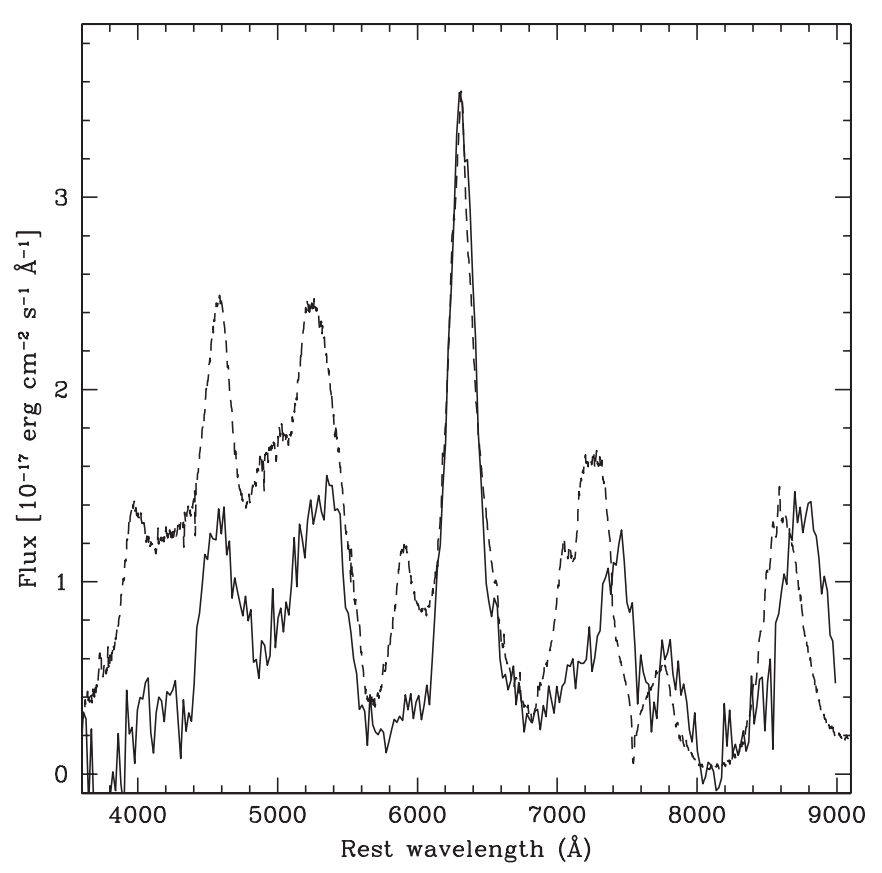

FIG. 2.- Spectrum of SN 2006aj obtained at the Keck Observatory on 2006 July 26, corresponding to a rest-frame epoch of 153 days after XRF 060218 (solid line), calibrated and binned with a $25 \AA$ boxcar, compared to the spectrum of SN 1998bw spectrum obtained on 1998 September 12 (Patat et al. 2001), at an epoch of 139 days after the explosion, which is assumed to have occurred on 1998 April 25, in coincidence with the GRB (dashed line). The flux of SN 1998bw has been rescaled to match that of SN 2006aj at the peak of the [O I] line. [See the electronic edition of the Journal for a color version of this figure.]

observed VLT spectrum, the fiducial galaxy spectrum, the observed spectrum recalibrated according to the galaxy photometry, as explained above, and the subtracted SN spectrum, calibrated and cleaned of cosmic rays.

\section{RESULTS}

Close inspection of the VLT FORS1 polarization data shows no detection of linear polarization, either in the continuum or in the most prominent emission line, [O I] $\lambda \lambda 6300,6363$; only a conservative $3 \sigma$ upper limit of $2 \%$ across the whole wavelength range could be set. Considering that a polarization level of $P \approx$ $1.4 \%$ may be attributed to the host galaxy (Gorosabel et al. 2006), and that interstellar polarization may contribute a similar amount $\left[P_{\mathrm{IS}, \max }=0.9 E(B-V)\right]$, this is consistent with unpolarized SN flux, and with a global ellipticity of the inner SN ejecta (i.e., at $v<8000 \mathrm{~km} \mathrm{~s}^{-1}$ ) of no more than $10 \%$ (Höflich 1991). This is not expected to be observable in the profiles of the nebular emission lines.

We derived SN magnitudes $B \approx 22.8, V \approx 21.6, R \approx 21.0$, and $I \approx 20.9$ for the Keck data (rest-frame epoch 153 days), and $B \approx 23.4, V \approx 22.6$, and $R \approx 21.8$ for the VLT data (rest-frame epoch 206 days). We assign an uncertainty of $\sim 0.1 \mathrm{mag}$ in the $R$, $V$, and $I$ bands, and $0.2 \mathrm{mag}$ in the $B$ band. The average lightcurve decline rate in this period is then $\sim 1.7 \pm 0.7,1.5 \pm 0.4$, and $1.2 \pm 0.3 \mathrm{mag}(100 \text { days })^{-1}$ in the $V, R$, and $I$ bands, respectively. This is consistent with expectation for a late-time light curve powered by gamma rays from ${ }^{56} \mathrm{Co}$ decay in relatively transparent SN ejecta and is also consistent with other SNe Ibc such as SN 1998bw (Patat et al. 2001) and SN 2002ap (Foley et al. 2003; Tomita et al. 2006).

The Keck spectrum, obtained on 2006 July 26, has a restframe epoch of 153 days. Figure 2 shows a comparison with the 


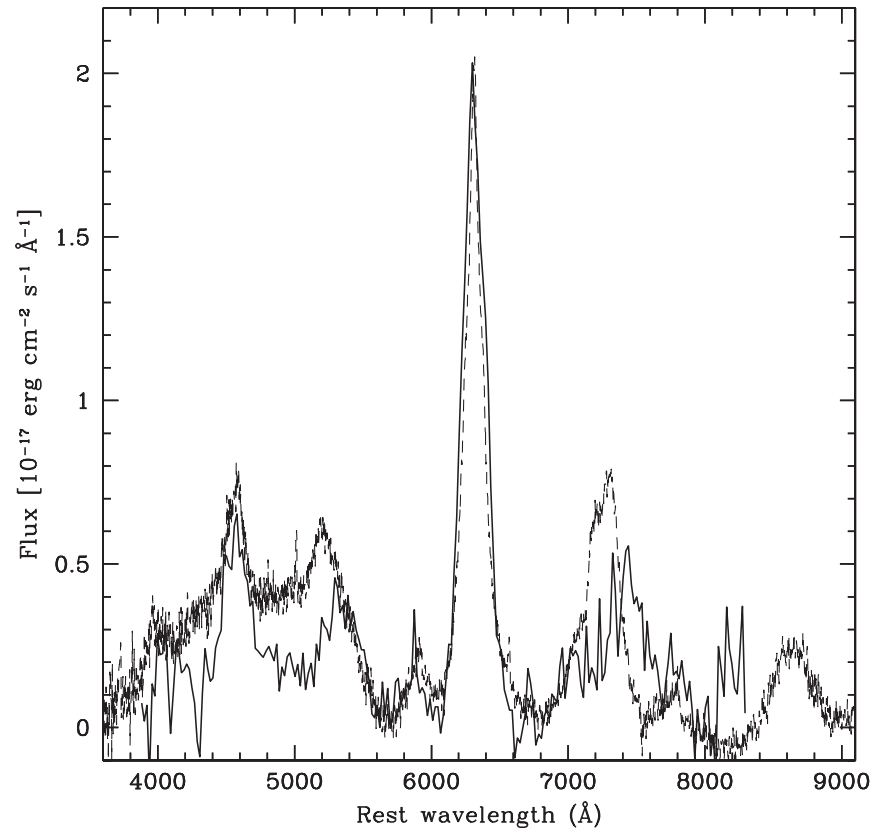

FIG. 3.-Comparison of the VLT 2006 September 19-20 (206 rest-frame days after XRF 060218) spectrum of SN 2006aj, calibrated and binned with a $20 \AA$ boxcar (solid line), with the SN 1998bw spectrum obtained on 1998 November 26 (Patat et al. 2001), 214 rest-frame days after GRB 980425 (dashed line). [See the electronic edition of the Journal for a color version of this figure.]

SN 1998bw spectrum obtained on 1998 September 12, at an epoch of 139 days after the explosion, which is assumed to have occurred on 1998 April 25, in coincidence with GRB 980425 (Pian et al. 2000). The two spectra are very similar, but obviously the $\left[\mathrm{O}_{\mathrm{I}}\right]$ line is stronger relative to all other lines in $\mathrm{SN}$ 2006aj.

The VLT spectrum, obtained on 2006 September 19-20, has a rest-frame epoch of 206 days. It is shown in Figure 3 compared to the spectrum of SN 1998bw obtained on 1998 November 26, at a rest-frame epoch of 216 days after the explosion. Again, [O I] $\lambda \lambda 6300,6363$ is by far the strongest emission line, and is stronger in SN 2006aj than in SN 1998bw relative to the other lines, indicating that oxygen dominates the composition of the ejecta. The next strongest lines are near $4600 \AA$, corresponding to $\mathrm{Mg} \mathrm{I}]$ $\lambda 4571 \AA$, and a line near $7400 \AA$. This is too red to be Ca II] $\lambda \lambda 7291,7324$, and [ $\mathrm{Ni}$ II] $\lambda 7380$ is the best candidate (Maeda et al. 2007). The wavelength of the emission near $8700 \AA$ in the Keck spectrum suggests that the Ca II IR triplet is not the only contributor, and that [C I] $\lambda 8727$ is also strong. The [Fe II] lines are relatively weak, but still form an emission feature near $5300 \AA$. The strongest lines in that complex have rest wavelengths of $5159,5262,5273$, and $5334 \AA$. [ Fe III] lines are very weak, as in all SN Ic spectra, indicating low temperature and significant clumping.

The profile of the emission lines in the nebular spectrum of SN 2006aj is not very sharp, indicating that the nebula is to a good approximation spherically symmetric. Figure 4 shows a comparison of the [O I] $\lambda \lambda 6300,6363$ line of $\mathrm{SNe} 1998 \mathrm{bw}$, 2006aj, and 2003jd. The line in SN 2006aj is broader than the other two by $\sim 2000 \mathrm{~km} \mathrm{~s}^{-1}$, although the ejecta velocity of SN 2006aj was significantly lower than that of SN 1998bw. The line of SN 1998bw is quite sharp, which was interpreted as a disklike distribution of slow-moving oxygen viewed from a near-polar direction (Maeda et al. 2002). In SN 2003jd the line has a width similar to that of SN $1998 \mathrm{bw}$, but the double-peaked profile sug-

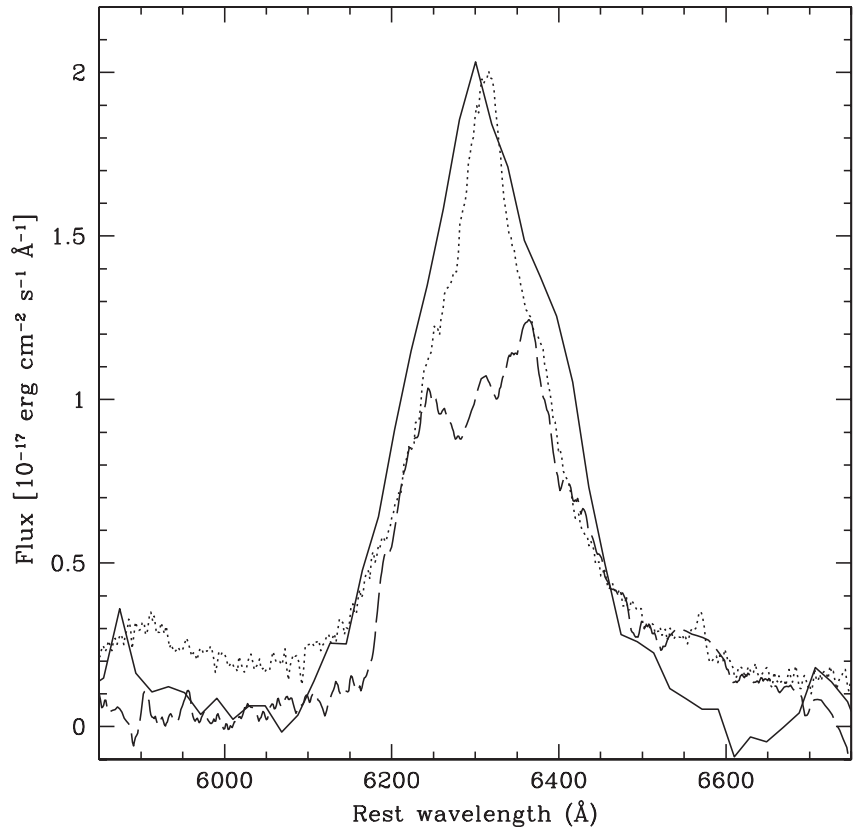

FIG. 4.-Comparison of the [O I ] $\lambda \lambda 6300,6363$ line of SNe 2006aj (solid line), 1998bw (dotted line; Patat et al. 2001), and 2003jd (dashed line; Mazzali et al. 2005). The profile of the line in SN 2006aj is less peaked than that of SN 1998bw, although the average expansion velocity of other elements is larger in SN 1998bw than in SN 2006aj, indicating a smaller degree of asphericity. [See the electronic edition of the Journal for a color version of this figure.]

gests that we are viewing the oxygen-rich disk from near its plane (Mazzali et al. 2006b).

\section{MODELS}

We modeled the nebular spectra using a non-LTE code (Mazzali et al. 2001) based on the approximations discussed in Axelrod (1980) and Ruiz-Lapuente \& Lucy (1992). The deposition of the gamma rays emitted in the decay of ${ }^{56} \mathrm{Co}$ to ${ }^{56} \mathrm{Fe}$ is computed using a gray opacity, while the positrons that are also produced are assumed to deposit their energy in situ. Collisional heating by the fast particles produced by the deposition of the gamma rays and the positrons is computed, and is balanced by cooling via line emission. The emission lines depend on the composition; in the case of a SN Ic, oxygen dominates the cooling.

For both spectra the spherical approximation seems reasonable, and so we use the code in its simplest version, assuming that the emitting volume is spherical and homogeneous. This allows us to test the basic geometric properties of the ejecta of SN 2006aj by verifying any deviation of the nebular lines from a parabolic profile. We adopted a distance of $140 \mathrm{Mpc}$ and a reddening correction of $E_{B-V}=0.13 \mathrm{mag}$ (Pian et al. 2006), which takes into account both Galactic and intrinsic absorption.

The main values of the fits are shown in Table 1 . The outer velocity of the model for the Keck spectrum is $8000 \mathrm{~km} \mathrm{~s}^{-1}$, while for the later VLT spectrum this value is $7400 \mathrm{~km} \mathrm{~s}^{-1}$. A slight decrease of the outer velocity may be expected, as the expansion makes the density decrease with time, such that outer regions may progressively become too thin for collisional excitation processes to work efficiently.

The Keck spectrum (Fig. 5) requires a ${ }^{56} \mathrm{Ni}$ mass of $0.20 \pm$ $0.01 M_{\odot}$, in excellent agreement with the early light-curve model (Mazzali et al. 2006b), and an oxygen mass of $1.50 \pm 0.15 M_{\odot}$. Uncertainties are estimated from parameter combinations that 
TABLE 1

Model Properties

\begin{tabular}{|c|c|c|c|c|c|c|c|}
\hline UT Date & $\begin{array}{c}\text { Epoch } \\
\text { (rest-frame days) }\end{array}$ & $\begin{array}{c}v \\
\left(\mathrm{~km} \mathrm{~s}^{-1}\right)\end{array}$ & $\begin{array}{c}M\left({ }^{56} \mathrm{Ni}\right) \\
\left(M_{\odot}\right)\end{array}$ & $\begin{array}{c}M_{\mathrm{ej}} \\
\left(M_{\odot}\right)\end{array}$ & $\begin{array}{l}M(\mathrm{O}) \\
\left(M_{\odot}\right)\end{array}$ & $\begin{array}{l}M(\mathrm{C}) \\
\left(M_{\odot}\right)\end{array}$ & $\begin{array}{c}L \\
\left(\operatorname{ergs~s}^{-1}\right)\end{array}$ \\
\hline 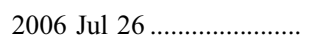 & 153 & 8000 & $0.20 \pm 0.01$ & $2.07 \pm 0.20$ & $1.50 \pm 0.15$ & $0.30 \pm 0.05$ & $1.3 \times 10^{41}$ \\
\hline
\end{tabular}

yield acceptable fits. The line near $7400 \AA$ is mostly due to [Ni II] 27380. Given the late epoch of the spectra, stable ${ }^{58} \mathrm{Ni}$ must be responsible for the emission, which can be reproduced assuming a ${ }^{58} \mathrm{Ni}$ mass of $0.02 M_{\odot}$. The consequences of this on the properties of the progenitor star are discussed in Maeda et al. (2007). Other lines contributing to the emission are [Fe II] $\lambda \lambda 7155,7453$ and [Co II] $\lambda 7541$. The $\mathrm{Ca}$ II] $\lambda \lambda 7291,7324$ lines are very weak. Similarly, the emission near $8600 \AA$ is mostly due to [C I] $\lambda 8727$, while the Ca II IR triplet is very weak. The line can be reproduced only if we assume the presence of $\sim 0.3 M_{\odot}$ of carbon. This is an interesting result, as in the early phase no carbon was visible in the spectrum (Mazzali et al. 2006b). The low calcium abundance is also interesting, as it may be a clue of the nucleosynthesis in the progenitor and in the explosion. The $\mathrm{Ca} / \mathrm{O}$ fraction is $\sim 10^{-4}$ by mass, whereas the corresponding value for SN 1998bw was $\sim 10^{-2}$. The total mass enclosed within the outer velocity of $8000 \mathrm{~km} \mathrm{~s}^{-1}$ is $2.07 \pm 0.20 M_{\odot}$.

The ${ }^{56} \mathrm{Ni}$ mass estimated for the VLT spectrum is $0.19 \pm$ $0.01 M_{\odot}$, and the oxygen mass is $1.42 \pm 0.15 M_{\odot}$ (Fig. 6). The total mass enclosed within the outer velocity of $7400 \mathrm{~km} \mathrm{~s}^{-1}$ is $1.94 \pm 0.25 M_{\odot}$. The slight reduction in ejected mass derived from the later VLT spectrum with respect to the earlier Keck spectrum is a consequence of the slightly narrower emission lines, and is consistent with the model used to fit the early light curve and spectra (Mazzali et al. 2006b). However, while the mass of ${ }^{56} \mathrm{Ni}$ derived from the nebular models $\left(\approx 0.20 M_{\odot}\right)$ is in excellent

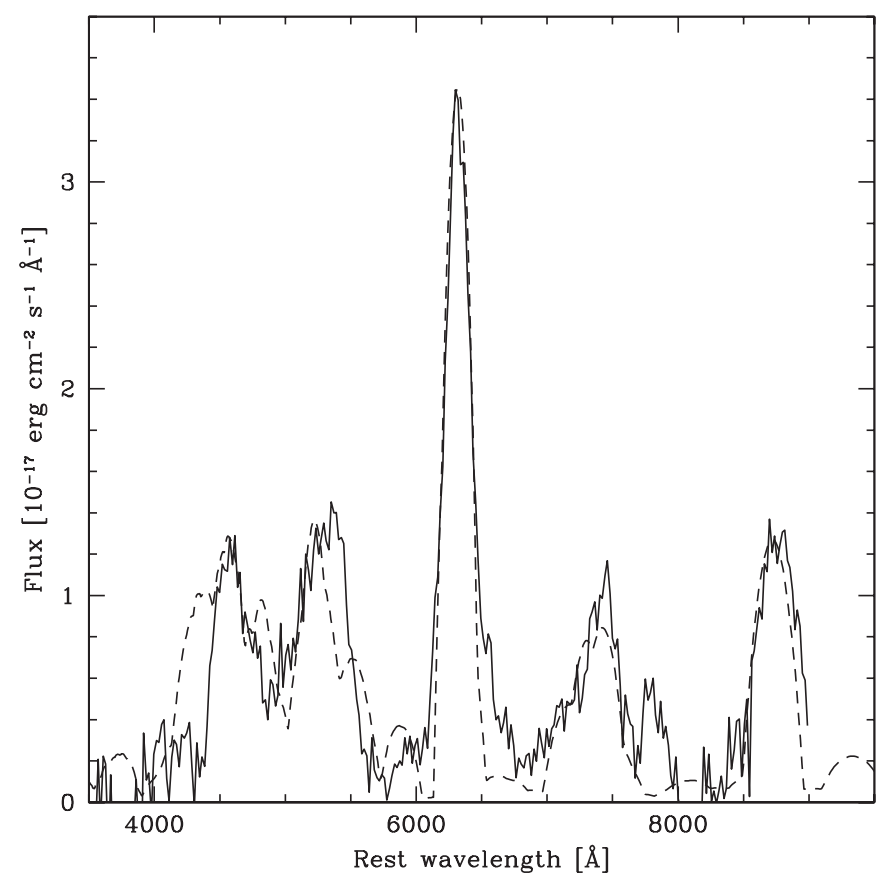

FIG. 5.-Keck spectrum of 2006 July 26 (solid line) and the corresponding synthetic spectrum (dashed line). [See the electronic edition of the Journal for a color version of this figure.] agreement with the value estimated from the early light-curve evolution, the ejected mass, and in particular the masses of oxygen and carbon, are larger.

The last early-time spectrum, obtained on 2006 March 10 (Pian et al. 2006), was modeled for a photospheric velocity of $10,000 \mathrm{~km} \mathrm{~s}^{-1}$, so there is no overlap between the region sampled by the early data (above $10,000 \mathrm{~km} \mathrm{~s}^{-1}$ ) and that sampled by the nebular spectra (below $8000 \mathrm{~km} \mathrm{~s}^{-1}$ ), except that the early light curve depends on the density of the innermost regions. The ejected mass predicted below $8000 \mathrm{~km} \mathrm{~s}^{-1}$ by the model used by Mazzali et al. $(2006 \mathrm{~b})$ is $\sim 1 M_{\odot}$, so the nebular results suggest the presence of a dense inner region containing an additional $\sim 1 M_{\odot}$ mostly composed of oxygen $\left(\sim 0.7 M_{\odot}\right)$ and carbon $\left(\sim 0.3 M_{\odot}\right)$, signs of which were not visible in the earlytime spectra. The presence of an inner high-density core is typical of other SNe Ic (e.g., SN 1998bw and SN 2002ap), as discussed in $\S 5$.

The only deviation from a parabolic profile may be seen in the core of the $\left[\mathrm{O}_{\mathrm{I}}\right]$ line. This may be due to the assumption of a constant density, or to the presence of an oxygen-dominated, highdensity inner core. The profile suggests that the oxygen abundance is dropping at velocities between 2000 and $4000 \mathrm{~km} \mathrm{~s}^{-1}$, but is higher again at the lowest velocities $\left(v \leq 2000 \mathrm{~km} \mathrm{~s}^{-1}\right)$, Between 2000 and $4000 \mathrm{~km} \mathrm{~s}^{-1}$ intermediate-mass elements may dominate, but the high flux at the lowest velocities may indicate the presence of a disklike oxygen-rich region. Further data and more detailed modeling will clarify this.

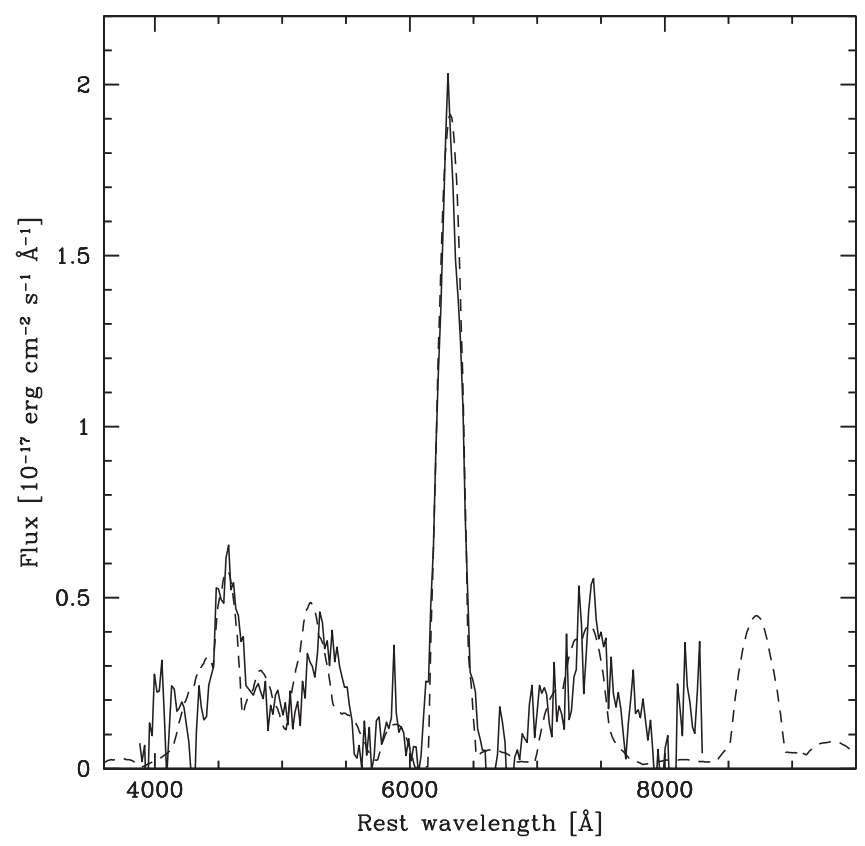

FIG. 6.- VLT spectrum of 2006 September 19-20 (solid line) and the corresponding synthetic spectrum (dashed line). [See the electronic edition of the Journal for a color version of this figure.] 


\section{DISCUSSION}

The nebular spectra of SN 2006aj are dominated by strong [O I] $\lambda \lambda 6300,6363$ emission, as expected for a SN Ic. The masses derived from the modeling are small, in general agreement with the values obtained from the early-phase modeling, but indicate the presence of a central low-velocity zone containing $\sim 1 M_{\odot}$ of mostly carbon and oxygen. The abundance of calcium is also very low in the nebula. The presence of a dense, oxygen-rich core has been deduced from the nebular spectra (SN 1998bw; Mazzali et al. 2001), or via the analysis of the light curves (Maeda et al. 2003) in all well-observed broad-lined SNe Ic, and it is a strong indication that the explosion was not spherically symmetric. Only if the mass of this zone is large can an effect on the early light curve be appreciated (e.g., SN 1997ef; Maeda et al. 2003). Unfortunately, in the case of SN 2006aj spectroscopic observations in the early phase extended only to 2006 March 10 ( 20 days after the explosion), and the evolution of the light curve at epochs of 2-3 months, when the linear decline is a signature of the inner dense zone (Maeda et al. 2003), was missed. Nevertheless, the power of a combined approach of studying both the early phase and the late phase is highlighted by the present results.

The nebular lines have rather symmetric profiles. In particular, the [O I] line is not as sharp as in SN 1998bw, indicating a low degree of asymmetry. Early radio observations suggested that any aspherical outflow was characterized by a large opening angle (Soderberg et al. 2006b), and our results confirm that the ejecta of SN 2006aj look more like those of ordinary SNe Ic (e.g., SN 1994I; Filippenko et al. 1995; Sauer et al. 2006) than those of SN 1998bw. Early-phase modeling suggested that only a small fraction of the ${ }^{56} \mathrm{Ni}$ synthesized by the $\mathrm{SN}$ was ejected at high velocities, and our nebular models confirm that most of the ${ }^{56} \mathrm{Ni}$ was ejected at low velocities.

The only possible deviation from sphericity may be in the deepest regions, as indicated by the low-velocity enhancement of the $\left[\mathrm{O}_{\mathrm{I}}\right]$ line. This may indicate an oxygen-rich disk viewed nearly face-on, as in SN 1998bw but of much smaller size, confirming that all $\mathrm{SNe}$ Ic are probably intrinsically aspherical in their cores (Leonard \& Filippenko 2005; Leonard et al. 2006). Given the small size of this region, and limited quality of our polarization data, we do not expect that we can detect polarization from that structure. In addition, at the late phases of these data, polarization is more sensitive to the distribution of ${ }^{56} \mathrm{Ni}$ than to global asymmetries of the ejecta. The lack of a detection of polarization may therefore also indicate that the ${ }^{56} \mathrm{Ni}$ distribution in the inner ejecta was not very aspherical.

Our results confirm that the mass ejected in the SN explosion was small, and thus lend credence to our earlier conclusion that the progenitor star had a ZAMS mass of $\sim 20 M_{\odot}$, and that probably the remnant was a neutron star (Mazzali et al. 2006b; see also Maeda et al. 2007). They may also shed some light on the magnetar phenomenon. If the birth of a magnetar was at the origin of the XRF, this magnetic activity did not lead to gross asphericity in the SN ejecta or to the synthesis of much ${ }^{56} \mathrm{Ni}$ at high velocities. Probably, it did not lead to a jet at all, but rather to broad polar outflows. This should be useful not only for further modeling, but also to improve our understanding of the nature of XRFs. As mentioned by Pian et al. (2006) XRFs may be intrinsically weaker and more spherical versions of the classical, highly collimated cosmological GRBs.

Continued monitoring of SN 2006aj is planned at both Keck and VLT. When more data are collected, we will study in detail the evolution of the spectra and the light curve in the late phase.

We thank the ESO Director for allocating time to this program. We also thank the night astronomers of the Paranal Science Operations Team, the staff of the Keck Observatory, and Dan Kocevski for help with the observations. This work was partially supported by the Italian Ministry for University and Research (MIUR) under COFIN 2004 "The Physics of the Explosion of Massive Stars." This work was partially conducted by the groups of J. S. B. and A. V. F. under US Department of Energy SciDAC grant DE-FC02-06ER41453. A. V. F. is also grateful for the support of NSF grant AST 06-07485.
Appenzeller, I. 1998, Messenger, 94, 1

Axelrod, T. S. 1980, Ph.D. thesis, Univ. California, Santa Cruz

Cool, R. J., et al. 2006, GCN Circ. 4777, http:/gen.gsfe.nasa.gov/gen/gen3/ 4777.gen 3

Filippenko, A. V. 1982, PASP, 94, 715 1997, ARA\&A, 35, 309

Filippenko, A. V., \& Leonard, D. C. 2004, in Cosmic Explosions in Three Dimensions, ed. P. Höflich, P. Kumar, \& J. C. Wheeler (Cambridge: Cambridge Univ. Press), 30

Filippenko, A. V., et al. 1995, ApJ, 450, L11

Foley, R. J., et al. 2003, PASP, 115, 1220

Fynbo, J. P. U., et al. 2004, ApJ, 609, 962

Galama, T. J., et al. 1998, Nature, 395, 670

Gorosabel, J., et al. 2006, A\&A, 459, L33

Greiner, J., et al. 2003, Nature, 426, 157

Heise, J., in't Zand, J., Kippen, R. M., \& Woods, P. M. 2001, in Gamma-Ray Bursts in the Afterglow Era, ed. E. Costa, F. Frontera, \& J. Hjorth (Berlin: Springer), 16

Höflich, P. 1991, A\&A, 246, 481

Horne, K. 1986, PASP, 98, 609

Iwamoto, K., et al. 1998, Nature, 395, 672

Kawabata, K., et al. 2002, ApJ, 580, L39 2003, ApJ, 593, L19

Leonard, D. C., \& Filippenko, A. V. 2005, in ASP Conf. Ser. 342, 1604-2004: Supernovae as Cosmological Lighthouses, ed. M. Turatto et al. (San Francisco: ASP), 330

Leonard, D. C., Filippenko, A. V., Chornock, R., \& Foley, R. 2002, PASP, 114, 1333

\section{REFERENCES}

Leonard, D. C., et al. 2006, Nature, 440, 505

MacFadyen, A. I., \& Woosley, S. E. 1999, ApJ, 524, 262

Maeda, K., Nakamura, T., Nomoto, K., Mazzali, P. A., Patat, F., \& Hachisu, I. 2002, ApJ, 565, 405

Maeda, K., Nomoto, K., Mazzali, P. A., \& Deng, J. 2006, ApJ, 640, 854

Maeda, K., et al. 2003, ApJ, 593, 931 2007, ApJ, 658, L5

Malesani, D., et al. 2004, ApJ, 609, L5

Matheson, T., Filippenko, A. V., Ho, L. C., Barth, A. J., \& Leonard, D. C. 2000 , AJ, 120,1499

Mazzali, P. A., Nomoto, K., Maeda, K., \& Patat, F. 2001, ApJ, 559, 1047

Mazzali, P. A., et al. 2003, ApJ, 599, L95

. 2005, Science, 308, 1284

- 2006a, ApJ, 645, 1323

2006b, Nature, 442, 1018

Modjaz, M., et al. 2006, ApJ, 645, L21

Oke, J. B., et al. 1995, PASP, 107, 375

Patat, F., \& Romaniello, M. 2006, PASP, 118, 146

Patat, F., et al. 2001, ApJ, 555, 900

Pian, E., et al. 2000, ApJ, 536, 778

. 2006, Nature, 442, 1011

Ramirez-Ruiz, E., et al. 2005, ApJ, 625, L91

Ruiz-Lapuente, P., \& Lucy, L. B. 1992, ApJ, 400, 127

Sauer, D. N., Mazzali, P. A., Deng, J., Valenti, S., Nomoto, K., \& Filippenko, A. V. 2006, MNRAS, 369, 1939

Soderberg, A. M., Nakar, E., Berger, E., \& Kulkarni, S. K. 2006a, ApJ, 638, 930

Soderberg, A. M., et al. 2005, ApJ, 627, 877 
Soderberg, A. M., et al. 2006b, Nature, 442, 1014

Sollerman, J., et al. 2006, A\&A, 454, 503

Stanek, K. Z., et al. 2003, ApJ, 591, L17

Tominaga, N., Deng, J., Mazzali, P. A., Maeda, K., Nomoto, K., Pian, E., Hjorth, J., \& Fynbo, J. P. U. 2004, ApJ, 612, L105
Tomita, H., et al. 2006, ApJ, 644, 400

Wade, R. A., \& Horne, K. 1988, ApJ, 324, 411

Wang, L., Baade, D., Höflich, P., \& Wheeler, J. C. 2003, ApJ, 592, 457

Wang, L., Wheeler, J. C., Li, Z., \& Clocchiatti, A. 1996, ApJ, 467, 435

Woosley, S. E., \& Bloom, J. S. 2006, ARA\&A, 44, 507 